\title{
The longest ever randomised controlled trial of insulin glargine: study design and $\mathrm{HbA}_{1 \mathrm{c}}$ findings
}

\author{
C. J. Currie
}

Received: 7 July 2009 / Accepted: 8 July 2009 /Published online: 7 August 2009

(C) Springer-Verlag 2009

\author{
Abbreviations \\ NPH Neutral protamine Hagedorn \\ OHA Oral hypoglycaemic agent \\ PDR Proliferative diabetic retinopathy
}

To the Editor: I would like to discuss the study design and reporting of the recent study by Rosenstock and colleagues that examined the long-term safety of insulin glargine (A21Gly,B31Arg,B32Arg human insulin) vs neutral protamine Hagedorn (NPH) insulin with respect to progression of retinopathy [1].

It is possible that the inclusion of individuals with type 2 diabetes who, at baseline, had a mean duration of prior exposure to insulin of 5 years, had an average duration of diabetes of almost 11 years, and had not developed anything more than non-proliferative retinopathy may have introduced bias in the form of a 'survival effect'. That is, these patients could be those least likely to develop more severe visual loss, irrespective of their treatment regimen. This would have reduced the power of the study to detect any change resulting from insulin glargine exposure. There appeared to be considerable treatment heterogeneity in the respective treatment arms. For example, the mean total insulin dose in these participants was not a sum of the basal

\section{J. Currie $(\square)$}

The Pharma Research Centre, Cardiff MediCentre,

School of Medicine, Cardiff University,

Cardiff CF14 4UJ, UK

e-mail: currie@cardiff.ac.uk and prandial components, suggesting that more detail would be helpful in understanding just how the two alternative insulin regimens and insulin/oral hypoglycaemic agent (OHA) combinations evolved within the trial. The authors did not report the use of OHAs at all, except for one remark in the discussion and a post hoc analysis, which was intended to explain the difference in $\mathrm{HbA}_{1 \mathrm{c}}$ levels; thus, treatment exposure patterns were not clear. Detailed reporting of concomitant metformin is particularly important with respect to the follow-up cancer risk data [2], since metformin is associated with almost complete attenuation of cancer risk [3]. We know that insulin glargine users are more likely than other insulin analogue users, including insulin detemir (NN304) [B29Lys( $\varepsilon$-tetradecanoyl),desB30 human insulin] users, to also receive metformin in a basal/ oral combination [4].

Although glycaemia $\left(\mathrm{HbA}_{1 \mathrm{c}}\right)$ was not the primary endpoint of this study [1], there was a small but statistically significant difference in mean $\mathrm{HbA}_{1 \mathrm{c}}$ between the two treatment arms, with the $\mathrm{HbA}_{1 \mathrm{c}}$ level $0.2 \%$ lower $(p=0.005)$ in the NPH treatment group at the end of what was the longest and largest randomised controlled study of insulin glargine to date. Details of this important finding were, unfortunately, excluded from the abstract of the manuscript. With regard to the specific objectives of this study, little attention was drawn to the difference in the 
relative likelihood of progressing to proliferative diabetic retinopathy (PDR). For insulin glargine, the rate of progression to PDR (relative to NPH insulin) was 1.52 using intention-to-treat data, and 1.38 using per protocol data (Table 3 in [1]). This trend deserved further comment. I would also like to see a post hoc, subgroup analysis of the number of patients with $a \geq 3$ step progression in the Early Treatment Diabetic Retinopathy Study (ETDRS) retinopathy severity scale score at endpoint, but only in those people with evidence of retinopathy at baseline. Akin to the safety issue with respect to cancer [5], it could be that insulin glargine does not trigger the pathology, but, rather, promotes or accelerates the pathological process.

To better understand these data, and to determine if in fact there was no increased risk of retinopathy within this trial, I suggest that an alternative, post hoc analysis of these data should be performed that compares the progression of visual morbidity in people treated with insulin glargine vs NPH insulin as a function of total insulin glargine exposure. I would be better convinced if it could be demonstrated that the risk of both progressive retinopathy (and cancer, for that matter) was the same after adjusting for baseline differences and for total, non-glargine insulin exposure (with or without metformin) in the following three cohorts: a group that received no insulin glargine (i.e. an NPH insulin group), and low and high insulin glargine exposure groups. Finally, a subgroup analysis of the $\mathrm{HbA}_{1 \mathrm{c}}$ data (Fig. 2b in [1]) was carried out (results presented in Fig. 2c in [1]). It would be useful to have this analysis replicated for hypoglycaemia data (Fig. 2d in [1]), as it is possible that this also explains this small difference in outcome between the two treatment arms.

This study was a key safety study. One of the most striking and important findings from this study was that the largest ever study characterising insulin glargine did not demonstrate improved glycaemic control over NPH insulin, and that any differences in glycaemia $\left(\mathrm{HbA}_{1 \mathrm{c}}\right)$ and in hypoglycaemia were probably due to differences in the combination insulin regimens and in concomitant OHA medication. Thus, in this study there was no treatment benefit from using insulin glargine over NPH insulin in the face of postulated safety concerns, not to mention its higher unit costs. It is in everyone's interest to finally put to bed any lingering concerns about the relative safety of insulin glargine. To be totally convincing with regard to progression of retinopathy (and cancer), further post hoc analyses of this particular study would be helpful, as would a metaanalysis of all appropriate randomised clinical trial data held by the manufacturer, and parallel studies of observational data.

Duality of interest The author declares that there is no duality of interest associated with this manuscript.

\section{References}

1. Rosenstock J, Fonseca V, McGill JB et al (2009) Similar progression of diabetic retinopathy with insulin glargine and neutral protamine Hagedorn (NPH) insulin in patients with type 2 diabetes: a long-term, randomised, open-label study. Diabetologia. doi:10.1007/s00125-009-1415-7

2. Rosenstock J, Fonseca V, McGill JB et al (2009) Similar risk of malignancy with insulin glargine and neutral protamine Hagedorn $(\mathrm{NPH})$ insulin in patients with type 2 diabetes: findings from a 5 year randomised, open-label study. Diabetologia. doi:10.1007/ s00125-009-1452-2

3. Currie CJ, Poole CD, Gale EAM (2009) The influence of glucoselowering therapies on cancer risk in type 2 diabetes. Diabetologia. doi:10.1007/s00125-009-1440-6

4. Poole CD, Tetlow T, McEwan P, Holmes P, Currie CJ (2007) The prescription cost of managing people with type 1 and type 2 diabetes following initiation of treatment with either insulin glargine or insulin detemir in routine general practice in the UK: a retrospective database analysis. Curr Med Res Opin 23(Suppl. 1): S41-S48

5. Smith U, Gale EAM (2009) Does diabetes therapy influence the risk of cancer? Diabetologia. doi:10.1007/s00125-009-1441-5 